\title{
CARACTERES MORFOMÉTRICOS EN DIFFLUGIA CORONA (TESTACEA, DIFFLUGIDAE) EN AMBIENTES LÉNTICOS DEL CHACO, ARGENTINA
}

\author{
Marcela Adriana Blanco ${ }^{1,2}$
}

\begin{abstract}
MORPHOMETRIC CHARACTERS OF DIFFLUGIA CORONA (TESTACEA, DIFFLUGIDAE) IN LENTIC ENVIRONMENTS OF CHACO, ARGENTINA. Morphometric characters of two populations of Difflugia corona Wallich, 1864 collected in two climatic stations (Spring, Autumn) in lentic environments of Chaco Province, Argentina, are studied. In the first climatic station it was registered specimens of bigger size; in the second, the size of the individuals was very below the minimum values registered. It is suggested a possible relationship between the size of the organisms and the availability of the inhabitable vegetable substratum.
\end{abstract}

KEYWORDS. Difflugia, morphometric characters, Chaco, Argentina.

\section{INTRODUCCIÓN}

Difflugia corona Wallich, 1864 es una ameba testácea frecuente, en Argentina, en el eupleuston de ambientes lénticos subtropicales. Esta especie se caracteriza por presentar una teca esférica o subesférica, con un seudostoma provisto de numerosos dientes (generalmente más de 12) formando una corona irregular y otorgándole un aspecto crenulado. En la base de la teca presenta un número variable de cuernos (6 a 10), cónicos y más o menos divergentes. Su teca es de naturaleza exógena, y las partículas que forman parte de la misma se mantienen unidas por un cemento amarronado que le otorga al conjunto un tinte oscuro. La taxonomía de las amebas testáceas se basa, precisamente, en la morfología y morfometría de las tecas para la identificación específica.

Se tuvo la oportunidad de observar la existencia de individuos de Difflugia corona con tamaños que diferían marcadamente respecto a la especie tipo, lo que planteó la posibilidad de realizar un estudio comparativo entre las poblaciones en función de caracteres morfométricos de las tecas en dos estaciones climáticas diferentes (primavera y otoño).

\section{MATERIAL Y MÉTODOS}

Las zonas muestreadas corresponden a cuerpos de agua lénticos con extensos manchones de vegetación flotante ubicados a los lados de la Ruta Nacional $N^{\circ} 16$ (provincia de Chaco, Argentina). Los muestreos fueron llevados a cabo en dos períodos diferentes (octubre de 1997 y abril de 1998); en cada uno de ellos se relevaron cinco cuerpos de agua, todos los cuales se ponen en contacto con el río Paraná en la época de crecientes. La zona muestreada se extiende entre los $27^{\circ} 25^{\prime}$ y $27^{\circ} 27^{\prime} \mathrm{S}-58^{\circ} 45^{\prime}$ y $58^{\circ} 52^{\prime} \mathrm{O}$.

En ambos períodos de muestreo, la vegetación estuvo constituida por Pistia stratiotes Linné (ampliamente predominante), Salvinia biloba Raddi emend. de la Sota, Azolla filiculoides Lamarck y Lemna sp. En el primer período prospectado (primavera), la carpeta vegetal cubrió la totalidad del espejo de agua, en tanto en el segundo (otoño) el sustrato se presentó en forma de manchones más dispersos, dejando gran parte del espejo de agua desprovisto de vegetación.

1. Cátedra Zoología Invertebrados I, Faculdad de Ciencias Naturales y Museo, Paseo de Bosque s/n, 1900 La Plata, Argentina

2. Becaria de Perfeccionamiento de la Comisión de Investigaciones Científicas (CIC). 
El material biológico, obtenido por lavado de la vegetación flotante y filtrado del sedimento con una red de plancton de $35 \mu \mathrm{m}$ de abertura de malla, fue llevado a un volumen de $50 \mathrm{ml}$ y fijado con formol al $4 \%$ y depositado en la Cátedra Zoología Invertebrados I, Facultad de Ciencias Naturales y Museo, Universidad Nacional de La Plata. Se extrajeron 200 individuos de D. corona, los cuales fueron montados en glicerina y medidos bajo microscopio óptico. Para todos los individuos fueron estimados los siguientes parámetros morfométricos: longitud y ancho de la teca, diámetro del seudostoma y número de cuernos. Se construyeron polígonos de frecuencia con el fin de demostrar la distribución de la longitud y ancho de la teca, diámetro del seudostoma y número de cuernos para cada uno de los grupos de individuos de D. corona en consideración.

Se aplicó el índice de correlación de Pearson para evaluar la interrelación entre el diámetro de la teca y el del seudostoma, y entre el diámetro y longitud de las tecas (SOKAL \& RoHLF, 1979). Las abreviaturas utilizadas fueron: $\mathrm{n}=$ número total de datos evaluados, $\mathrm{CNS}=$ curva normal estándar.

En cada cuerpo de agua en estudio y con auxilio de un aparato de medición múltiple Corning® se obtuvieron datos de temperatura del agua, $\mathrm{pH}$, conductividad y concentración de oxígeno disuelto.

\section{RESULTADOS Y DISCUSIÓN}

En el primer período analizado (1997) la temperatura del agua osciló entre $17,5^{\circ} \mathrm{C}$ y $21,1^{\circ} \mathrm{C}$ con un promedio de $19,6^{\circ} \mathrm{C}$; los valores de $\mathrm{pH}$ fueron similares entre los distintos puntos registrándose valores muy próximos a la neutralidad. Las mayores diferencias se dieron en la conductividad, que varió entre $125 \mu$ Mhos y $600 \mu$ Mhos. En cuanto al oxígeno disuelto, se registraron aguas desde pobremente oxigenadas con valores de $0,8 \mathrm{mg} / \mathrm{l}$, hasta aquellas con concentraciones de $9 \mathrm{mg} / \mathrm{l}$. En 1998, los valores de $\mathrm{pH}$ oscilaron entre 7,33 y 8,33 , correspondiendo a ambientes neutros a moderadamente alcalinos. Nuevamente, las mayores diferencias se dieron con relación a la conductividad que varió de $70 \mu$ Mhos a 741,6 $\mu$ Mhos. Respecto al oxígeno disuelto, el ambiente se caracterizó por presentar aguas moderadamente oxigenadas.

Tabla I. Estadísticos correspondientes a Difflugia corona (primavera), en Chaco, Argentina, octubre de 1997.

\begin{tabular}{lrrrrr}
\hline & Longitud $(\mu \mathrm{m})$ & Ancho $(\mu \mathrm{m})$ & Seudostoma $(\mu \mathrm{m})$ & $\mathrm{N}^{\circ}$ de cuernos & $\mathrm{A} / \mathrm{Ps}$ \\
\hline $\mathrm{n}$ & 100,00 & 100,00 & 100,00 & 100,00 & 100,00 \\
Media & 159,00 & 162,70 & 56,60 & 3,34 & 3,02 \\
Desvío & 23,76 & 25,18 & 16,64 & 1,49 & 0,63 \\
Error estándar & 2,37 & 2,51 & 1,66 & 0,14 & 0,06 \\
Valor máximo & 220,00 & 230,00 & 90,00 & 7,00 & 5,33 \\
Valor mínimo & 100,00 & 100,00 & 30,00 & 0,00 & 1,00 \\
Lím. sup. 95\% & 163,75 & 167,73 & 59,92 & 3,63 & 3,14 \\
Lím. inf. 95\% & 154,24 & 157,66 & 53,27 & 3,04 & 2,87 \\
Lím. sup. 99\% & 166,12 & 170,25 & 61,59 & 3,78 & 3,21 \\
Lím. inf. 99\% & 151,87 & 155,14 & 51,60 & 2,89 & 2,81 \\
\hline
\end{tabular}

Tabla II. Estadísticos correspondientes a Difflugia corona (otoño), en Chaco, Argentina, abril de 1998.

\begin{tabular}{lrrrrr}
\hline & Longitud $(\mu \mathrm{m})$ & Ancho $(\mu \mathrm{m})$ & Seudostoma $(\mu \mathrm{m})$ & $\mathrm{N}^{\circ}$ de cuernos & A / Ps \\
\hline $\mathrm{n}$ & 100,00 & 100,00 & 100,00 & 100,00 & 100,00 \\
Media & 80,10 & 81,20 & 36,50 & 3,33 & 2,26 \\
Desvío & 15,47 & 17,24 & 7,57 & 1,14 & 0,40 \\
Error estándar & 1,54 & 1,72 & 0,75 & 0,11 & 0,04 \\
Valor máximo & 120,00 & 130,00 & 60,00 & 6,00 & 3,25 \\
Valor mínimo & 50,00 & 50,00 & 20,00 & 0,00 & 1,40 \\
Lím. sup. 95\% & 83,19 & 84,64 & 38,01 & 3,55 & 2,34 \\
Lím. inf. 95\% & 77,00 & 77,75 & 34,98 & 3,10 & 2,18 \\
Lím. sup. 99\% & 84,74 & 86,37 & 38,77 & 3,67 & 2,38 \\
Lím. inf. 99\% & 75,45 & 76,02 & 34,22 & 2,98 & 2,14 \\
\hline
\end{tabular}



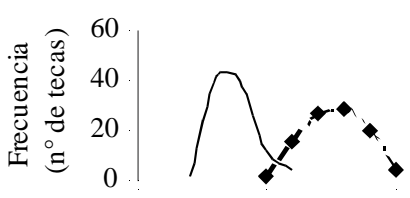

0

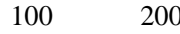

Longitud
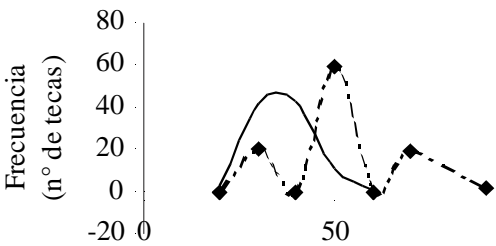

Diámetro del seudostoma
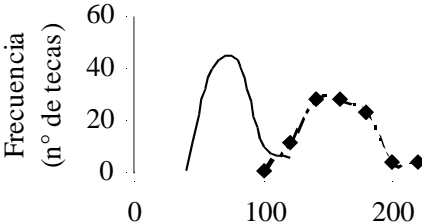

Ancho

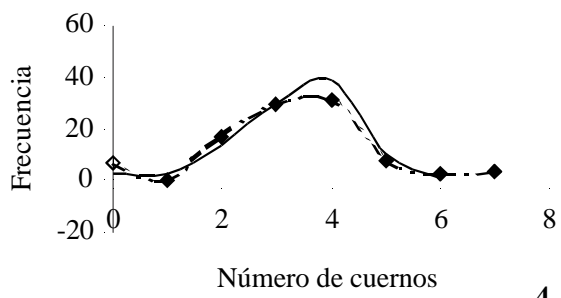

Figs. 1-4. Curvas de frecuencia de los parámetros morfométricos de primavera (línea quebrada) y de otoño (línea entera) de Difflugia corona, en Chaco, Argentina, octubre de 1997 y abril de 1998: 1, variación en la longitud de las tecas; 2, variación en el ancho de las tecas; 3, variación en el diámetro del seudostoma; 4, variación en el número de cuernos.

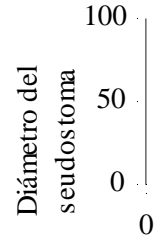

0

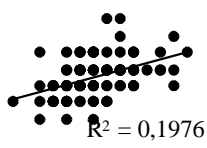

100
200

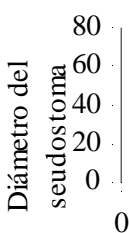

5

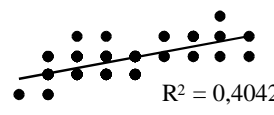

50
150

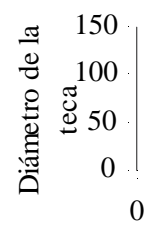

7

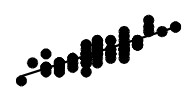

$R^{2}=0,7145$

$100 \quad 200$

Longitud de la teca
Diámetro de la teca

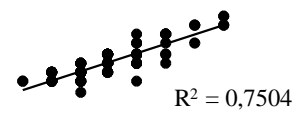

50

100

150

Longitud de la teca 8

Figs. 5-8. Difflugia corona em Chaco, Argentina. 5,6, Correlación entre diámetro de la teca y diámetro del seudostoma: 5, octubre de 1997 (primavera); 6, abril de 1998 (otoño); 7,8, Correlación entre diámetro y longitud de las tecas: 7, octubre de 1997 (primavera); 8, abril de 1998 (otoño). 
En la primavera, la longitud de las tecas varió de $100 \mu \mathrm{m}$ a $220 \mu \mathrm{m}$, el ancho de $100 \mu \mathrm{m}$ a $230 \mu \mathrm{m}$ y el diámetro del seudostoma de $30 \mu \mathrm{m}$ a $90 \mu \mathrm{m}$. En cuanto al número de cuernos, se registraron desde organismos carentes de ellos hasta aquellos con un número máximo de siete (tab. I). En los individuos del otoño la longitud osciló entre 50 $\mu \mathrm{m}$ y $120 \mu \mathrm{m}$, el ancho de $50 \mu \mathrm{m}$ a $130 \mu \mathrm{m}$, el diámetro del seudostoma de $20 \mu \mathrm{m}$ a 60 $\mu \mathrm{m}$ y el número de cuernos de 0 a 6 (tab. II).

En el análisis de frecuencia de los parámetros morfométricos evaluados (fig. 1) las curvas exhiben una distribución normal. Existen dos picos en la curva de distribución de la longitud, ancho de la teca y diámetro del seudostoma, lo cual pone en evidencia una clara separación de las poblaciones de primavera y otoño, cuyas medias difieren significativamente. Respecto del número de cuernos, en cambio, ambas poblaciones se solapan.

Comparando las poblaciones mediante el método de la CNS y para un nivel de significación $\alpha=0,05$ se concluye que, con relación a la longitud, ancho de la teca y diámetro del seudostoma, existen diferencias significativas en los caracteres morfométricos de las poblaciones de ambas estaciones climáticas. En cuanto al número de cuernos, las diferencias no son significativas.

Los tamaños registrados para la especie en otoño presentan un mínimo muy inferior a las dimensiones observadas por otros autores: LEIDY (1879) menciona tecas de $320 \mu \mathrm{m}$ de diámetro, en tanto PENARD (1902) señala diámetros de 200-250 $\mu \mathrm{m}$. Los diámetros de los especímenes de África dados por GAUTHIER-Lièvre \& Thomas (1958) varían de 120 $\mu \mathrm{m}$ a $190 \mu \mathrm{m}$. GREEN (1963) encontró individuos de esta especie en el río Sokoto (Nigeria, África) con un diámetro tecal que osciló entre $90 \mu \mathrm{m}$ a $125 \mu \mathrm{m}$, y fueron consideradas como "distinctly smaller than is usual for this species" (GREEN, 1963:504).

El diámetro de la teca y el del seudostoma (fig. 2) están estrechamente correlacionados en otoño $(r=0,63)$. Las tecas de tamaño pequeño tienen un diámetro promedio del seudostoma menor que el de aquellas de mayor porte; en primavera esta relación es no significativa. En tanto, como puede observarse (fig. 3), existe una marcada correlación entre el diámetro y longitud de la teca en ambas estaciones climáticas $\left(\mathrm{r}_{\text {primavera }}\right.$ $\left.=0,84 \mathrm{y} \mathrm{r}_{\text {otoño }}=0,86\right)$.

La vegetación de los cuerpos de agua está condicionada fundamentalmente por las fluctuaciones del nivel de las aguas, lo cual determina una desigual distribución específica de la vegetación. Precisamente, la menor representatividad de la carpeta vegetal durante la estación otoñal coincidió con la creciente del río Paraná que alcanzó una altura de 7,37 metros ("El Diario", 12 de abril de 1998, p. 4, Resistencia, Chaco, Argentina) en tanto en primavera, cuando el nivel del río osciló entre 4-5 metros, la vegetación cubrió por entero el cuerpo de agua.

NeIFF (1978) distingue crecientes "ordinarias" y crecientes "extraordinarias" o "extraordinariamente excepcionales" en el ciclo hidrológico anual del Paraná. Las primeras son crecientes de primavera-verano, en las cuales sus caudales alcanzan los $25.000 \mathrm{~m}^{3} \mathrm{~s}^{-1}$; las otras se producen generalmente cada cinco a diez años y llegan a superar los $40.000 \mathrm{~m}^{3} \mathrm{~s}^{-1}$. Durante estas últimas, se evidencia "un crecimiento rápido del cuerpo vegetativo de las plantas arraigadas emergentes, así como la dispersión, traslación y reacomodación de las plantas flotantes las que, conforme a la intensidad de la creciente, pueden ser eliminadas o prosperar en áreas más favorables".

Los parámetros físico-químicos, con sólo ligeras variantes, se mantuvieron dentro del rango en el cual $D$. corona puede desarrollarse con éxito. Los únicos cambios 
observados lo fueron en el hábitat, atribuyéndose a las intensas precipitaciones que afectaran al ambiente en primavera, dando lugar a una disminución o quizá eliminación y posterior reemplazo de la vegetación acuática. Se observó una disminución en el tamaño de las tecas concomitante con la reducción del sustrato disponible. Puede considerarse la disminución de tamaño de estos organismos como una modificación adaptativa? Al existir una disminución de la disponibilidad del hábitat, las formas más pequeñas y por lo tanto más livianas, tendrían mayores posibilidades de permanecer flotando en superficie, tal como sucede con los habitantes del plancton.

Agradecimientos. A la Dra. Estela C. Lopretto (Facultad de Ciencias Naturales y Museo, UNLP, La Plata, Argentina), por su apoyo permanente, lectura crítica del manuscrito y sus valiosos consejos. A la Dra. María C. Vucetich (UNLP), por contribuir con su asesoramiento y el aporte desinteresado de su vasta experiencia.

\section{REFERENCIAS BIBLIOGRÁFICAS}

Gauthier-Lièvre, L. \& Thomas, R. 1958. Les genres Difflugia, Pentagonia, Maghrebia et Hoogenraadia (Rhizopodes testacés) en Afrique. Arch. Protistenk., Jena, 103 (1-2): 241-370.

GreEn, J. 1963. Zooplankton of the river Sokoto. The Rhizopoda Testacea. Proc. zool. Soc. Lond., London, 141 (3):497-514.

LEIDY, J. 1879. Freshwater rhizopods of North America. Rep. U. S. Geol. Surv. Terr., Washington, 12:1-324. NeIFF, J. J. 1978. Fluctuaciones de la vegetación acuática en ambientes del valle de inundación del Paraná medio.

Physis, Buenos Aires, 38(95):41-53.

Penard, E. 1902. Faune rhizopodique du bassin du Léman. Genève, Henry Kündig. 714p.

SоKAL, R. R. \& RoHLF, F. J. 1979. Biometría. Principios y métodos estadísticos en la investigación biológica. Madrid, Blume. $832 \mathrm{p}$.

Recebido em 10.08.2000; aceito em 30.03.2001 